\title{
An alternative dynamic fundamental solution for plate bending including the shear deformation effect
}

\author{
L. Palermo Jr. \\ Faculty of Civil Engineering, Architecture and Urban Design, \\ State University of Campinas, Brazil
}

\begin{abstract}
The elastodynamic solution for plate bending including the shear deformation effect is modified in the present study. A regular potential function at the source point was adopted in the fundamental solution for the unit couple as the main change with reference to the former solution. The use of regular functions does not affect the switching of the solution including effects of shear deformation and rotatory inertia to that for the classical model when variables related to them are turned null. Furthermore, the present function in the vector potential field is reduced to that employed in static analysis when the frequency value is zero. The main benefit in the present formulation is the reduction in the number of points employed in numerical integrations. Results obtained for the first natural frequency are compared to available solutions in the literature to show the behavior of the present formulation.

Keywords: harmonic solution, bending including the shear deformation effect, the Mindlin bending model.
\end{abstract}

\section{Introduction}

The plate bending model including the effect of shear deformation is very efficient when is necessary to evaluate stress concentration in the edge zone of the plate or around holes that have a diameter not larger than eight times of the plate thickness [1] whereas the overall plate behavior can be analyzed with the classical bending model. Reissner [2] first presented a model to consider the effect of shear deformation in static analyses. The discrepancies in dynamic analysis using the classical model with reference to three-dimensional theory 
were shown by Mindlin [3] who presented a plate bending model similar to that proposed by Reissner and included the influence of rotatory inertia to perform dynamic analysis. Years later, Mindlin has shown in the study of flexural vibration of rectangular plates [4] that three types of vibration modes could be identified when the effect of shear deformation was considered: the flexural, the thickness-shear and the thickness-twist mode. The decoupling of the direct relation between values of deflection derivatives and plate rotations was the main consequence from combination of those vibration modes in general vibration analyses. When the vibration modes were analyzed independently, the direct relation between values of plate rotation and deflection derivative appeared in the flexural mode but not in the thickness-shear mode. On the other hand, the thickness-twist mode had only plate rotations, i.e. the model where the complete decoupling was identified. The frequencies related to the thickness-twist mode were called breathing frequencies by Levinson [5] who pointed out on difficulties to identify frequencies according to the thickness-shear mode in general vibration analyses.

The effects of shear deformation and rotatory inertia could be considered alone or coupled in the elastodynamic formulation presented in [6], which was not the same presented in [7] where only the real part of the solution was adopted and a limit for angular frequencies had to be introduced. The present study improved the elastodynamic solution [6] by considering a regular potential function at the source point in fundamental solutions for unit couples. The particular solutions related to unit couples were added in both potential fields (scalar and vector) of the elastodynamic solution in other strategy with reference to [8]. Terms related to particular solutions in [8] disappeared in the plate rotations vector due to the use of the field decomposition and were disregard in the final expressions of potentials fields in the former elastodynamic solution [6].

The solution due to unit load remained unchanged in the present formulation whereas solutions due to unit couples were modified by the addition of the particular solution to the solution of Helmholtz's equation in each potential function. The use of regular functions do not affect the switching of the solution including effects of shear deformation and rotatory inertia to that for the classical model when variables related to them are turned null. An interesting feature appeared in the function of the vector potential field, which is reduced to that employed in static analysis [9] when the frequency value is zero.

The numerical implementation employed quadratic shape functions for isoparametric boundary elements with collocation points always placed on the boundary. The reduction of the number of points employed in numerical integrations was the good feature resultant from the use of regular functions. Results obtained for the first natural frequency are compared to available solutions in the literature beyond to those obtained with the solution presented in [6] to show the behavior of the present formulation. 


\section{Alternative elastodynamic solution}

The equations of motion in time-harmonic problems for an infinitesimal plate element under a transverse distributed loading $\mathrm{q}\left(\mathrm{t}, \mathrm{x}_{\mathrm{i}}\right)$ are next written with Latin indices taking values $\{1,2$ and 3$\}$ and Greek indices taking values $\{1,2\}$

$$
\begin{gathered}
M_{\alpha \beta, \beta}-Q_{\alpha}=\frac{\rho h^{3}}{12} \frac{\partial^{2} \psi_{\alpha}}{\partial t^{2}} \\
Q_{\alpha, \alpha}+q=\rho h \frac{\partial^{2} w}{\partial t^{2}}
\end{gathered}
$$

The plate has a uniform thickness $\mathrm{h}$ and the mass density $\rho$. The transverse acceleration $\partial^{2} \mathrm{w} / \partial \mathrm{t}^{2}$ and the angular acceleration $\partial^{2} \psi_{\alpha} / \partial \mathrm{t}^{2}$ are functions of the deflection $\mathrm{w}$ and plate rotations $\psi_{\alpha}$, respectively. The constitutive relations are next written

$$
\begin{gathered}
M_{\alpha \beta}=D \frac{(1-v)}{2}\left(\psi_{\alpha, \beta}+\psi_{\beta, \alpha}+\frac{2 v}{1-v} \psi_{\gamma, \gamma} \delta_{\text {á } \beta}\right) \\
Q_{\alpha}=D \frac{(1-v)}{2} \lambda^{2}\left(\psi_{\alpha}+w_{, \alpha}\right)
\end{gathered}
$$

with

$$
\lambda^{2}=12 \frac{\kappa^{2}}{h^{2}}
$$

$\mathrm{D}$ is the flexural rigidity, $v$ is the Poisson ratio. The shear parameter $\kappa^{2}$ can be a fixed value equal to $\pi^{2} / 12$ or a function of the Poisson ratio according to [3], which is given by

$$
4 \sqrt{\left(1-\frac{(1-2 v)}{2(1-v)} \kappa^{2}\right)\left(1-\kappa^{2}\right)}=\left(2-\kappa^{2}\right)^{2}
$$

The decomposition of the plate rotations vector $\left(\psi_{\alpha}\right)$ in the scalar $\left(\phi_{\alpha}\left(\mathrm{x}_{\alpha}, \omega\right)\right)$ and the vector potential field $\left(0,0, \mathrm{H}\left(\mathrm{x}_{\alpha}, \omega\right)\right)$ remained, as done in fundamental solutions presented in $[6,8,9]$

$$
\psi_{\alpha}=\frac{\partial}{\partial x_{\alpha}}\left[\phi_{1}\left(\delta_{1} r\right)+\phi_{2}\left(\delta_{2} r\right)\right]+e_{3 \alpha \beta} \frac{\partial}{\partial x_{\beta}}\left[H\left(\delta_{3} r\right)\right]
$$

$e_{3 \alpha \beta}$ is the permutation symbol. The arguments of the potential functions are dependent of variables related to the rotatory inertia $(\mathrm{R})$, the shear deformation (S) and the classical dynamic factor for plates $\left(\delta_{0}^{4}\right)[3]$

$$
\begin{gathered}
\delta_{1}=\frac{1}{2} \delta_{0}^{4}\left(R+S+\sqrt{(R-S)+\frac{4}{\delta_{0}^{4}}}\right) ; \quad \delta_{2}=\frac{1}{2} \delta_{0}^{4}\left(R+S-\sqrt{(R-S)+\frac{4}{\delta_{0}^{4}}}\right) \\
\delta_{3}=\frac{2}{1-v}\left(R \delta_{0}^{4}-\frac{1}{S}\right)=\lambda^{2}\left(R S \delta_{0}^{4}-1\right) \\
R=\frac{h^{2}}{12} ; \quad S=\frac{1}{\lambda^{2}} \frac{2}{(1-v)} ; \quad \delta_{0}^{4}=\frac{\omega^{2} \rho h}{D}
\end{gathered}
$$

The relation between the deflection and scalar potential functions $\left(\phi_{\alpha}\right)[6,8]$ remained

and

$$
w=-\left(\beta_{1}+1\right) \phi_{1}\left(\delta_{1} r\right)-\left(\beta_{1}+1\right) \phi_{2}\left(\delta_{2} r\right)
$$

$$
\beta_{1}=S\left(\delta_{1}^{2}-\delta_{0}^{4} R\right) ; \quad \beta_{2}=S\left(\delta_{2}^{2}-\delta_{0}^{4} R\right)
$$


The fundamental solution can be written in terms of Hankel functions of the first kind or modified Bessel functions with complex arguments [6, 8]. The expressions for potential functions in the scalar and the vector potential field are next written in terms of modified Bessel functions:

a) The solution due to the unit point force is the same present in [6]

$$
\phi_{1}=-\frac{1}{2 \pi D} \frac{1}{\left(\delta_{1}^{2}-\delta_{2}^{2}\right)} K_{0}\left(i \delta_{1} r\right) ; \quad \phi_{2}=\frac{1}{2 \pi D} \frac{1}{\left(\delta_{1}^{2}-\delta_{2}^{2}\right)} K_{0}\left(i \delta_{2} r\right) ; \quad H=0
$$

b) The solution due to unit couple in direction $\gamma$ has the particular solution added to the solution of Helmholtz's equation in each potential function

$$
\begin{gathered}
\phi_{1}=-\frac{1}{2 \pi D} \frac{1}{\left(\delta_{1}^{2}-\delta_{2}^{2}\right)} \frac{\partial}{\partial x_{\gamma}}\left[K_{0}\left(i \delta_{1} r\right)+\ln (r)\right] ; \\
\phi_{2}=\frac{1}{2 \pi D} \frac{1}{\left(\delta_{1}^{2}-\delta_{2}^{2}\right)} \frac{\partial}{\partial x_{\gamma}}\left[K_{0}\left(i \delta_{2} r\right)+\ln (r)\right] ; \\
H=-e_{3 \gamma \eta} \frac{1}{2 \pi D} \frac{1}{\delta_{3}^{2}} \frac{2}{(1-v)} \frac{\partial}{\partial x_{\eta}}\left[K_{0}\left(i \delta_{3} r\right)+\ln (r)\right]
\end{gathered}
$$

The present elastodynamic solution becomes equal to that obtained for the classical bending model [10] when variables related to effects of shear deformation $(\mathrm{S})$ and rotatory inertia $(\mathrm{R})$ are set equal to zero. The plate rotations turn directly defined from the deflection function derivatives as result from the classical model because constants $\beta_{\alpha}$ are equal to zero. The solenoidal field $(\mathrm{H})$ must be set equal to zero in absence of the effect shear deformation according to the equations of motion for the classical model when they are written in terms of potentials. On the other hand, when the effect of shear deformation is considered and the frequency is set equal to zero, $\delta_{3}$ turns equal to $-\lambda^{2}$ and the potential function $\mathrm{H}$ becomes equal to that used in static analysis [9]. The reason of this feature is related to the differential equation to obtain the potential function $\mathrm{H}$ that remains unchanged in static analysis. Reissner first noted in [11] on the similarity between the differential equation related to the effect of shear deformation and the "wave" equation.

The modified Bessel functions with complex arguments $(\mathrm{z})$ can be written in terms of Bessel functions of the first $(\mathrm{J})$ and the second kind $(\mathrm{N})$ as can be done for Hankel functions [12]

$$
K_{\eta}(z)=\frac{\pi}{2} i^{\eta}\left[i J_{\eta}(i z)-N_{\eta}(i z)\right]
$$

The expansion of those Bessel functions with complex arguments around the origin, i.e. when $\mathrm{z}$ approaches to zero [12] shows

$$
\begin{gathered}
J_{\eta}(z) \rightarrow\left(\frac{1}{2} z\right)^{\eta} \frac{1}{\Gamma(\eta+1)} \\
N_{0}(z) \rightarrow \frac{2}{\pi} \ln z \\
N_{\eta}(z) \rightarrow-\frac{\Gamma(\eta)}{\pi}\left(2 \frac{1}{z}\right)^{\eta}
\end{gathered}
$$

The particular solution introduced in each potential function of the fundamental solution due to unit couple regularizes the potential function when $\mathrm{z}$ approaches to zero according to equations (12) to (14).

The static-like form of the equations of motion for harmonic problems led to a displacement boundary integral equation for the Mindlin model, similar to that 
presented for static problems [9] but used in conjunction with the elastodynamic fundamental solution instead

$$
\begin{gathered}
\frac{1}{2} C_{i j}\left(x^{\prime}\right) u_{j}\left(x^{\prime}\right)+\int_{\Gamma}\left[T_{i j}\left(x^{\prime}, x\right) u_{j}(x)-U_{i j}\left(x^{\prime}, x\right) t_{j}(x)\right] d \Gamma(x) \\
=\iint_{\Omega} U_{i 3}\left(x^{\prime}, X\right) q(X) d \Omega(X)
\end{gathered}
$$

$\mathrm{C}_{\mathrm{ij}}$ is an element of the matrix $\mathrm{C}$ related to the boundary at the source point, which becomes the identity matrix when a smooth boundary is considered. $\mathrm{u}_{\alpha}$ is $\psi_{\alpha}, \mathrm{u}_{3}$ is $\mathrm{w}, \mathrm{t}_{\alpha}$ is the product $\mathrm{M}_{\alpha \beta} \cdot \mathrm{n}_{\beta}, \mathrm{t}_{3}$ is the product $\mathrm{Q}_{\alpha} \cdot \mathrm{n}_{\alpha} \cdot \mathrm{U}_{\mathrm{ij}}$ represents the rotation $(\mathrm{j}=1,2)$ or the deflection $(\mathrm{j}=3)$ due to a unit couple $(\mathrm{i}=1,2)$ or a unit point force $(\mathrm{i}=3) . \mathrm{T}_{\mathrm{ij}}$ represents the moment $(\mathrm{j}=1,2)$ or the shear $(\mathrm{j}=3)$ due to a unit couple $(\mathrm{i}=1,2)$ or a unit point force $(\mathrm{i}=3)$.

The singularities in fundamental solution kernels of DBIE for harmonic loads, equation (15), are the same type of those in static DBIE [9] when the present elastodynamic solution is used, as summarized in Table 1.

Table 1: $\quad$ Singularity type using the present elastodynamic solution.

\begin{tabular}{|c|c|c|c|c|}
\hline Solution due to & $\begin{array}{c}\text { Deflection } \\
\mathrm{U}_{\mathrm{i} 3}\end{array}$ & $\begin{array}{c}\text { Rotation } \\
\mathrm{U}_{\mathrm{i} 2} \text { or } \mathrm{U}_{\mathrm{i} 3}\end{array}$ & $\begin{array}{c}\text { Shear } \\
\mathrm{T}_{\mathrm{i} 3}\end{array}$ & $\begin{array}{c}\text { Moment } \\
\mathrm{T}_{\mathrm{i} 2} \text { or } \mathrm{T}_{\mathrm{i} 3}\end{array}$ \\
\hline Unit Load $(\mathrm{i}=3)$ & Weak & Regular & Cauchy & Weak \\
\hline Unit Couple $(\mathrm{i}=1,2)$ & Regular & Weak & Weak & Cauchy \\
\hline
\end{tabular}

The expansion of Bessel functions in the neighborhood of the origin [12], equations (12) to (14), was used to obtain the behavior of fundamental solution kernels summarized in Table 1 . The weak type and the Cauchy type singularity are related to logarithmic singularity and $\mathrm{r}^{-1}$ singularity type, respectively.

\section{Numerical implementation}

The numerical implementation employed quadratic shape functions for isoparametric boundary elements with collocation points always placed on the boundary. The same mapping function was used for conformal and nonconformal interpolations, i.e. nodes at ends of quadratic elements remain at ends when discontinuous elements were employed and only the positions of collocation points were shifted. The collocation points were placed at nodes in case of continuous elements and at positions $(-0.67,0.0,+0.67)$, in the range $(-1,1)$, in case of discontinuous elements, i.e. the collocation points were shifted to inside the element at the corresponding end where the discontinuity exists. The singularity subtraction [13] and the transformation of variable technique [14] were employed for the Cauchy and the weak type singularity, respectively, when integrations were performed on elements containing the collocation points. The standard Gauss-Legendre scheme was employed for integrations on elements not containing the collocation points. A uniform load equal to $150 \mathrm{~N} \cdot \mathrm{m}^{-2}$ was applied on the plate surface, the Young modulus was $206.9 \mathrm{GPa}$, the Poisson ration $(v)$ was 0.3 , the mass density $(\rho)$ was $7860 \mathrm{~kg} \cdot \mathrm{m}^{-3}$, the side of 
the plate (a) had a length equal to $50 \mathrm{~cm}$ and the analysis used some values for the thickness (h). The ratio $\mathrm{h} / \mathrm{a}$ was varied from 0.05 to 1.0 so that, although the smallest value of $h / a$ is for a thin plate, for the larger values of $h / a$ one is considering blocks rather than plates. The solutions were compared to those available in the literature using the Mindlin model [4], the three-dimensional elasticity theory [5] and with the former solution [6].

The shear parameter $\kappa^{2}$ was equal to $\pi^{2} / 12$ to match the value used by Mindlin in [4]. The analyzed square plates were simply supported from all sides and the hard restraint condition was employed (the twisting moments $M_{n s}$ were restrained on the boundary) because this condition was used in $[4,5]$. The first natural angular frequency was identified using a harmonic excitation in the range up to $\delta_{0}^{2}$ equal to $12 \mathrm{a}^{-2}$ or $16 \mathrm{a}^{-2}$. The numerical solutions were evaluated using 20 incremental steps. In spite of the whole plate was modeled, the value of plate rotation at the center in the quarter of the plate was used in the analysis. The plate rotation values were used in the scanning because they appear in all vibration modes. A first evaluation was carried to find the focused interval. The focused interval containing 2 incremental steps was picked up when the signal of the real part of the value changes or a peak appears in the imaginary part of the value as shown in Figure 1, according to $[6,7]$. The focused interval was analyzed again using a step refinement and 20 incremental steps were used to scan this interval. Thus, two peaks with opposite signals could be identified in the response curve of the real part of the plate rotation as shown in [7]. An example of the frequency response for the plate rotation is next presented for $\mathrm{h} / \mathrm{a}$ equals to 0.2 .

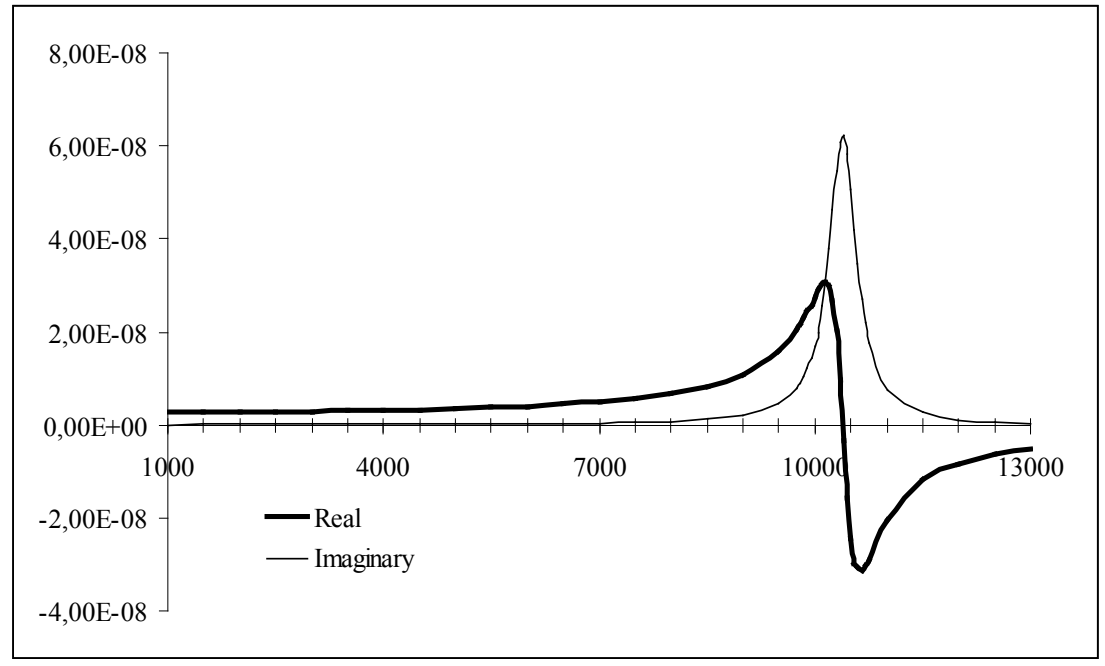

Figure 1: Frequency response for $\mathrm{h} / \mathrm{a}=0.2$ with rotatory inertia and shear.

The responses of the real and the imaginary parts for rotations or deflection values have the same aspect shown in Figure 1 for other ratios h/a. 
The first natural angular frequencies identified in the frequency response graphs are listed in Table 2 according to $\mathrm{h} / \mathrm{a}$ ratio. The frequency value corresponding to the peak of the imaginary part of the value for rotation was listed. It is necessary to point out that arguments $\mathrm{i}_{2}$ and $\mathrm{i} \delta_{3}$ have positive real values for ratios $\mathrm{h} / \mathrm{a}$ in the range 0.05 to 0.8 and then they become complexvalued for the ratio equal to 1.0 .

Table 2: Frequencies with the shear deformation and the rotatory inertia effects.

\begin{tabular}{|c|c|c|c|c|c|c|}
\hline $\begin{array}{c}\text { Ratio } \\
\text { h/a }\end{array}$ & $\begin{array}{c}\text { Elasticity } \\
{[4]}\end{array}$ & $\begin{array}{c}\text { Mindlin } \\
{[5]}\end{array}$ & {$[6]$} & Obtained & $\begin{array}{c}\text { Dif. to [6] } \\
(\%)\end{array}$ & $\begin{array}{c}\text { Dif. to [5] } \\
(\%)\end{array}$ \\
\hline 0.05 & 3016 & 3049 & 3015 & 3015 & 0,00 & -1.12 \\
\hline 0.1 & 5906 & 5918 & 5830 & 5800 & -0.51 & -1.99 \\
\hline 0.2 & 10880 & 10820 & 10360 & 10180 & -1.74 & -5.91 \\
\hline 0.4 & 17315 & 17073 & 15740 & 15200 & -3.43 & -10.97 \\
\hline 0.6 & 20734 & 20306 & 18960 & 18260 & -3.69 & $-10,08$ \\
\hline 0.8 & 22653 & 22079 & 21500 & 20750 & -3.49 & -6.02 \\
\hline 1.0 & 23805 & 23125 & 23450 & 23800 & +1.49 & +2.92 \\
\hline
\end{tabular}

The values obtained with the present formulation were close to expected values from [4-6] and the greatest differences to [5] (11\%) were obtained for ratios $\mathrm{h} / \mathrm{a}$ equal to 0.4 and 0.6 . A similar study was carried out in [15] with the boundary collocation method but there was used a soft restraint condition instead $\left(\mathrm{M}_{\mathrm{nt}}\right.$ was released). The differences between the former [6] and the present formulation were less than $5 \%$. The whole plate was modeled with continuous boundary elements along plate sides but two nodes were introduced at corners, i.e. the first and last boundary element in each side were mixed type (continuous at one end and discontinuous at other end). Results obtained with the former solution used 128 linear elements (132 nodes) and 16 Gauss points in numerical integrations whereas 32 quadratic elements (68 nodes) and 12 Gauss points were used with the present formulation. Furthermore, no selective integration was employed to obtain the results in this paper, i.e. a different number of Gauss points according to the distance to the collocation points was not used. There were tested other meshes with the present formulation:

a) 64 quadratic elements (132 nodes) and 12 Gauss Points;

b) 64 quadratic elements (132 nodes) and 6 Gauss Points;

c) 128 quadratic elements (260 nodes) and 6 Gauss Points.

The results were not changed with other meshes. The author believes the present solution becomes stable when 68 nodes were used. The differences between boundary element solutions were very low, i.e. values obtained with the former [6] the present formulation. The differences between Mindlin values and boundary element solutions can be attributed to a different shape of the vibration mode in the boundary element method with reference to that used by Mindlin where trigonometric functions were adopted as explained in [6]. 
The frequency response $(\mathrm{h} / \mathrm{a}=0.2)$ with linear elements [6], quadratic elements (132 nodes) with 12 and 6 Gauss points are shown in Figure 2. No difference appears in curves with quadratic elements using 12 and 6 Gauss points in numerical integration, i.e. values with 12 and 6 Gauss points were coincident.

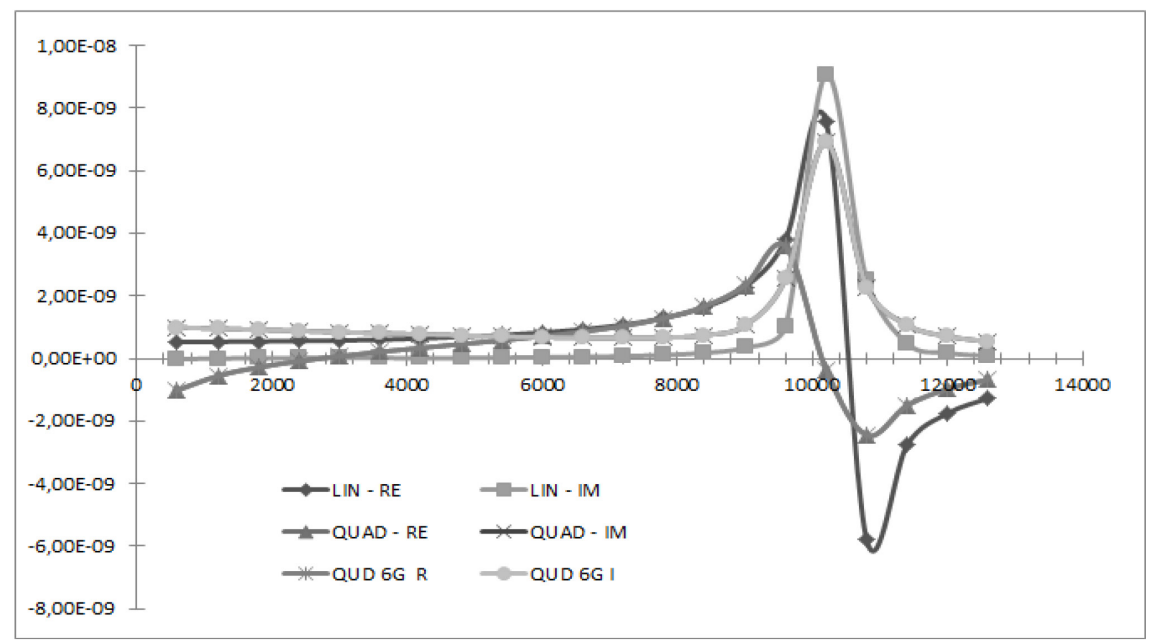

Figure 2: $\quad$ Frequency response with linear and quadratic elements.

The frequency response $(\mathrm{h} / \mathrm{a}=0.2)$ with quadratic elements but comparing results employing 68 nodes (12 Gauss points) with those employing 260 nodes (6 Gauss points) are shown in Figure 3. No difference appears in curves with 68 and 260 nodes. The curves for the real and the imaginary part of values for rotation were drawn, only.

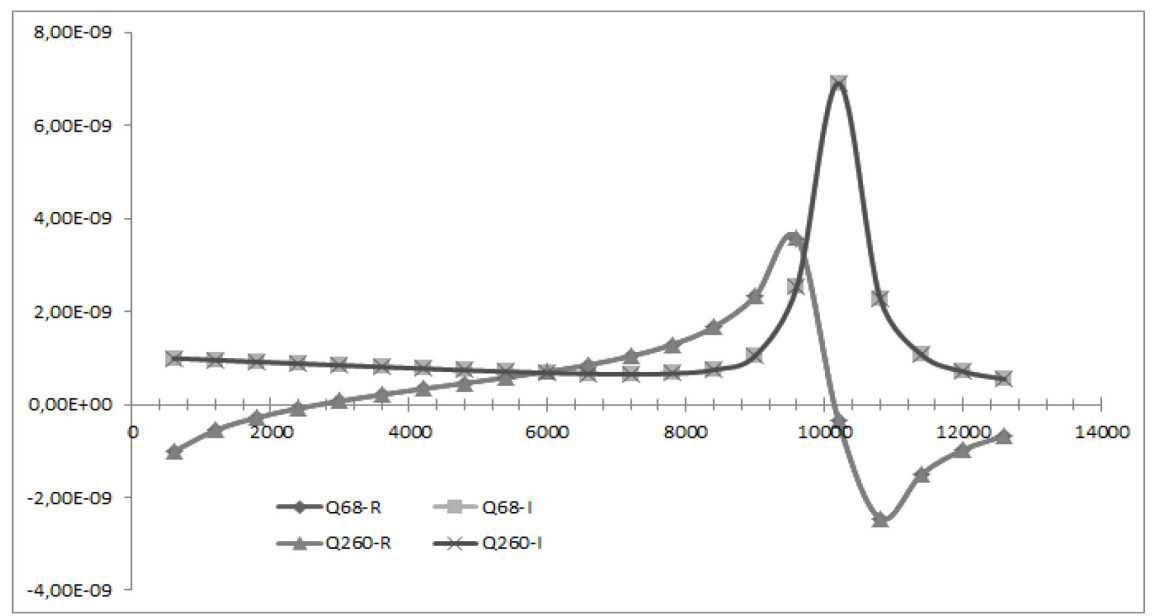

Figure 3: Frequency response with quadratic elements: 68 and 260 nodes. 


\section{Conclusions}

The present formulation improves the elastodynamic fundamental solution presented in [6]. The expressions for displacements (deflection and rotations) and tractions (moments and shear) contain singularity types in the same order of the static solution. In spite the introduction of the particular solutions in this study has handled with geometric singularity (ln $r$ ), the complete fundamental solution is better to satisfy the non-homogeneous differential equation containing singularities due to unit couples. The results obtained from the numerical implementation were very close to those obtained with [6] as well as to those in the literature $[4,5]$. Furthermore, reductions in the number of boundary nodes (degrees of freedom) and in the number of Gauss Points were identified in the analysis shown. Thus, the present formulation could be interesting in elastodynamic solutions requiring a lot of computation time to analyze complex engineering problems.

\section{Acknowledgements}

The author is grateful to CNPq and FAPESP for supporting the development of the research on plates.

\section{References}

[1] Timoshenko, S. P., Woinowsky-Krieger, S., Theory of Plates and Shells, McGraw-Hill Book Company, New York, 2nd Ed., 1959.

[2] Reissner, E., The Effect of Transverse Shear Deformation on the Bending of Elastic Plates, Journal of Applied Mechanics, 1945.

[3] Mindlin, R.D., Influence of rotatory inertia and shear on flexural motions of isotropic elastic plates, Journal of Applied Mechanics, 1951.

[4] Mindlin, R.D., Schacknow A., Deresiewicz, H., Flexural vibrations of rectangular plates, Journal of Applied Mechanics, 23, 430-436, 1956.

[5] Levinson, M., Free vibrations of a simply supported rectangular plate: an exact elasticity solution, Journal of Sound and Vibration, 98(2), 289-298, 1985.

[6] Palermo Jr., L., On the harmonic response of plates with the shear deformation effect using the elastodynamic solution in the boundary element method, Engineering Analysis with Boundary Elements, 2007.

[7] Antes, H., Static and Dynamic Analysis of Reissner-Mindlin Plates, Boundary Element Analysis of Plates and Shells, Editor: D.E. Beskos, Springer Series in Computational Mechanics Edited by S.N. Atluri, Springer-Verlag, 1991.

[8] Palermo Jr., L., On the fundamental solution to perform the dynamic analysis of Reissner-Mindlin's plates, Boundary Element Technology XV, Editors C.A. Brebbia, R.E. Dippery, 2003.

[9] Palermo Jr., L., Plate Bending analysis using the classical or the ReissnerMindlin models, Engineering Analysis with Boundary Elements, 2003. 
[10] Vivoli J, Filippi P. Eigenfrequencies of thin plates and layer potentials. J. Acoust. Soc. Am.; 55, 562-7, 1974.

[11] Reissner, E., On Bending of Elastic Plates, Q. Appl. Math., 1947.

[12] Abramowitz M, Stegun I. Handbook of mathematical functions, New York: Dover Publications; 1972.

[13] Aliabadi, M.H., The boundary element method, applications in solids and structures, vol.2, John Wiley and Sons Ltd., 2002.

[14] Telles, J.C.F, A self-adaptive coordinate transformation for efficient numerical evaluation of general boundary element integrals, Int. J. Num. Meth. Eng., 24, 959-973, 1987.

[15] Hutchinson, J.R., Analysis of plates and shells by boundary collocation, Editor: D.E. Beskos, Springer Series in Computational Mechanics Edited by S.N. Atluri, Springer-Verlag, 1991.

[16] Timoshenko S, Young, D.H., Weaver Jr., W., Vibration Problems in Engineering, $4^{\text {th }}$ ed., New York, John Wiley and Sons Ltd., 1974. 